ELECTRONIC RESEARCH ANNOUNCEMENTS OF THE AMERICAN MATHEMATICAL SOCIETY

Volume 2, Number 1, August 1996

\title{
GEODESIC LENGTH FUNCTIONS AND TEICHMÜLLER SPACES
}

\author{
FENG LUO
}

(Communicated by Walter Neumann)

\begin{abstract}
Given a compact orientable surface $\Sigma$, let $\mathcal{S}(\Sigma)$ be the set of isotopy classes of essential simple closed curves in $\Sigma$. We determine a complete set of relations for a function from $\mathcal{S}(\Sigma)$ to $\mathbf{R}$ to be the geodesic length function of a hyperbolic metric with geodesic boundary on $\Sigma$. As a consequence, the Teichmüller space of hyperbolic metrics with geodesic boundary on $\Sigma$ is reconstructed from an intrinsic combinatorial structure on $\mathcal{S}(\Sigma)$. This also gives a complete description of the image of Thurston's embedding of the Teichmüller space.
\end{abstract}

\section{$\S 1$. INTRODUCTION}

Let $\Sigma=\Sigma_{g, r}$ be a compact oriented surface of genus $g$ with $r$ boundary components. The Teichmüller space of isotopy classes of hyperbolic metrics with geodesic boundary on $\Sigma$ is denoted by $T(\Sigma)$, and the set of isotopy classes of essential simple closed unoriented curves in $\Sigma$ is denoted by $\mathcal{S}=\mathcal{S}(\Sigma)$. For each $m \in T(\Sigma)$ and $\alpha \in \mathcal{S}(\Sigma)$, let $l_{m}(\alpha)$ be the length of the geodesic representing $\alpha$.

An interesting question is to characterize the geodesic length functions among all functions defined on $\mathcal{S}(\Sigma)$. We announce in this note a solution to this question.

The solution is expressed in terms of an intrinsic combinatorial structure on $\mathcal{S}(\Sigma)$

Before stating the theorem, let us consider three basic examples which motivate the solution. We denote the isotopy class of a curve $s$ by $[s]$, and $\{t \in \mathbf{R} \mid t>a\}$ by $\mathbf{R}_{>a}$.

Example 1. If the surface is the three-holed sphere $\Sigma_{0,3}$, then the set $\mathcal{S}\left(\Sigma_{0,3}\right)$ consists of three elements which are the isotopy classes of the three boundary components of the surface. It is well known from the work of Fricke-Klein [FK] that any positive function from $\mathcal{S}(\Sigma)$ to $\mathbf{R}_{>0}$ is the geodesic length function of a unique element in the Teichmüller space.

For the rest of the note, we introduce the trace function $t_{m}(\alpha)=2 \cosh l_{m}(\alpha) / 2$ from $\mathcal{S}(\Sigma)$ to $\mathbf{R}_{>2}$. We will deal with the trace function $t_{m}$ instead of $l_{m}$.

Example 2. The surface is the one-holed torus $\Sigma_{1,1}$. Let $\mathcal{S}^{\prime}$ be the set $\{[s] \in \mathcal{S} \mid$ $s$ is not homotopic to the boundary $\left.\partial \Sigma_{1,1}\right\}$. It is well known that $\mathcal{S}^{\prime}$ is in oneone correspondence with the set of rational numbers $\mathbf{Q} \cup\{\infty\}$ where the map

Received by the editors April 9, 1996.

1991 Mathematics Subject Classification. Primary 32G15, 30F60.

Key words and phrases. Hyperbolic metrics, geodesics, Teichmüller spaces.

(C)1996 American Mathematical Society 


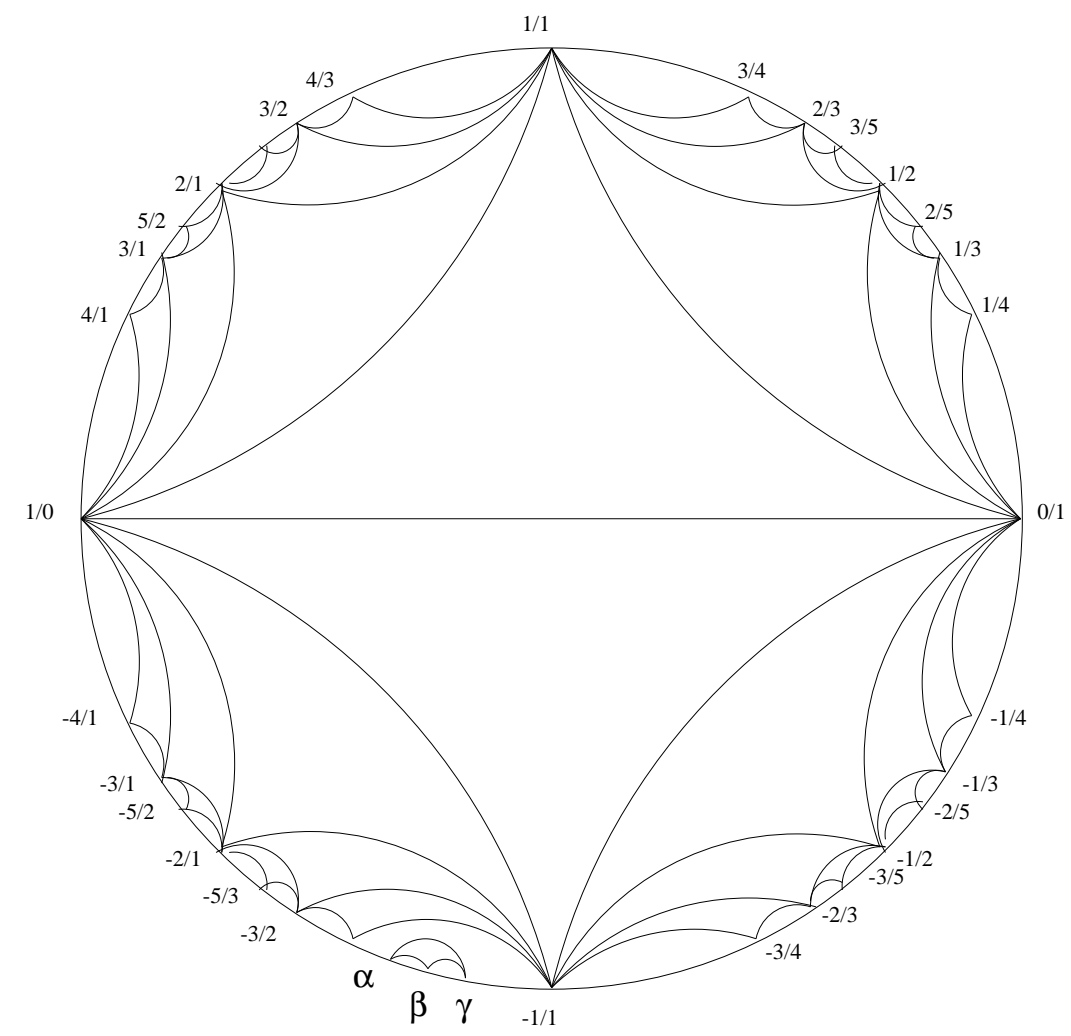

FiguRe 1

sends the isotopy class $[s]$ to the "slope" of $[s]$. Two rational numbers $p / q$ and $p^{\prime} / q^{\prime}$ satisfying $p q^{\prime}-p^{\prime} q= \pm 1$ correspond to two isotopy classes which contain two simple closed curves $a$ and $b$ intersecting at one point transversely. Thus the modular configuration comes in as a combinatorial structure on $\mathcal{S}^{\prime}$. A result of Fricke-Klein proved by L. Keen $[\mathrm{Ke}]$ gives a solution to the characterization problem. Namely, a function $f: \mathcal{S}\left(\Sigma_{1,1}\right) \rightarrow \mathbf{R}_{>2}$ is the trace $t_{m}$ for some $m$ in the Teichmüller space if and only if

$$
\begin{gathered}
f(\alpha) f(\beta) f(\gamma)+2=f^{2}(\alpha)+f^{2}(\beta)+f^{2}(\gamma)+f\left(\left[\partial \Sigma_{1,1}\right]\right), \\
f(\gamma)+f\left(\gamma^{\prime}\right)=f(\alpha)+f(\beta),
\end{gathered}
$$

where $(\alpha, \beta, \gamma)$ and $\left(\alpha, \beta, \gamma^{\prime}\right)$ are two ideal triangles in the modular configuration.

Example 3. The surface is the four-holed sphere $\Sigma_{0,4}$. Let $\mathcal{S}^{\prime}$ be the set $\{[s] \in \mathcal{S} \mid$ $s$ is not homotopic to the boundary $\left.\partial \Sigma_{0,4}\right\}$. It is well known [De], [Th] that $\mathcal{S}^{\prime}$ is in one-one correspondence with the set of rational numbers $\mathbf{Q} \cup\{\infty\}$ so that two rational numbers $p / q$ and $p^{\prime} / q^{\prime}$ satisfy $p q^{\prime}-p^{\prime} q= \pm 1$ if and only if the isotopy classes $p / q$ and $p^{\prime} / q^{\prime}$ are distinct and contain two simple closed curves $a$ and $b$ which intersect at two points. Thus again the modular configuration comes in as a combinatorial structure on $\mathcal{S}^{\prime}$. It is shown in [Lu1] that a function $f: \mathcal{S}\left(\Sigma_{0,4}\right) \rightarrow$ 
$\mathbf{R}_{>2}$ is the trace $t_{m}$ for some $m$ in the Teichmüller space if and only if

$$
\begin{aligned}
f(\alpha) f(\beta) & f(\gamma)+4 \\
= & f^{2}(\alpha)+f^{2}(\beta)+f^{2}(\gamma)+f(\alpha)\left(f\left(b_{i}\right) f\left(b_{j}\right)+f\left(b_{k}\right) f\left(b_{l}\right)\right) \\
& +f(\beta)\left(f\left(b_{i}\right) f\left(b_{k}\right)+f\left(b_{j}\right) f\left(b_{l}\right)\right)+f(\gamma)\left(f\left(b_{i}\right) f\left(b_{l}\right)+f\left(b_{j}\right) f\left(b_{k}\right)\right) \\
& +\sum_{s=1}^{4} f^{2}\left(b_{s}\right)+f\left(b_{1}\right) f\left(b_{2}\right) f\left(b_{3}\right) f\left(b_{4}\right), \quad i \neq j \neq k \neq i, \\
& f(\gamma)+f\left(\gamma^{\prime}\right)=f(\alpha) f(\beta)-f\left(b_{i}\right) f\left(b_{l}\right)-f\left(b_{j}\right) f\left(b_{k}\right)
\end{aligned}
$$

where $(\alpha, \beta, \gamma)$ and $\left(\alpha, \beta, \gamma^{\prime}\right)$ are two ideal triangles in the modular configuration, and $b_{s}$ 's $(s=1,2,3,4)$ are the isotopy classes of the four boundary components of $\partial \Sigma_{0,4}$ so that each of the triples $\left\{\alpha, b_{i}, b_{j}\right\},\left\{\beta, b_{i}, b_{k}\right\}$ and $\left\{\gamma, b_{i}, b_{l}\right\}$ bounds a 3-holed sphere in $\Sigma$.

Our main result states that relations $(1),\left(1^{\prime}\right),(2)$ and $\left(2^{\prime}\right)$ are the set of all relations for a function from $\mathcal{S}(\Sigma)$ to $\mathbf{R}_{>2}$ to be the trace function $t_{m}$ for an element $m$ in the Teichmüller space.

To be more precise, we introduce a combinatorial structure (corresponding to the modular configuration) on $\mathcal{S}$ as follows. Given two isotopy classes $\alpha$ and $\beta$, let $\mathrm{I}(\alpha, \beta)$ be the geometric intersection number between $\alpha$ and $\beta$ in $\mathcal{S}(\Sigma)$, i.e., $\mathrm{I}(\alpha, \beta)$ $=\operatorname{Min}\{|a \cap b| \mid a \in \alpha$ and $b \in \beta\}$, where $|a \cap b|$ is the number of points in $a \cap b$. If two simple closed curves $a$ and $b$ intersect at one point transversely (resp. $\alpha$, $\beta \in \mathcal{S}(\Sigma)$ with $\mathrm{I}(\alpha, \beta)=1$ ), we denote it by $a \perp b$ (resp. $\alpha \perp \beta$ ); if two simple closed curves $a$ and $b$ intersect at two points of different signs transversely and $\mathrm{I}([a],[b])=2$, we denote it by $a \perp_{0} b$. In this case, we denote the relation between their isotopy classes by $[a] \perp_{0}[b]$. Suppose $x$ and $y$ are two $\operatorname{arcs}$ in $\Sigma$ so that $x$ intersects $y$ transversely at one point. Then the resolution of $x \cap y$ from $x$ to $y$ is defined as follows. Take any orientation on $x$ and use the orientation on $\Sigma$ to determine an orientation on $y$. Now resolve the intersection point $x \cap y$ according to the orientations (see Figure 2(a)). If $a \perp b$ or $a \perp_{0} b$, we define $a b$ to be the curve obtained by resolving intersection points in $a \cap b$ from $a$ to $b$. If $\alpha \perp \beta$ or $\alpha \perp_{0} \beta$, we define $\alpha \beta$ to be $[a b]$ where $a \in \alpha$ and $b \in \beta$ with $|a \cap b|=\mathrm{I}(\alpha, \beta)$. Note that in the examples 2 and $3,\left(\gamma, \gamma^{\prime}\right)$ is $(\alpha \beta, \beta \alpha)$.

Theorem. For a surface $\Sigma_{g, r}$ of negative Euler number, a function l from $\mathcal{S}\left(\Sigma_{g, r}\right)$ to $\mathbf{R}_{t>0}$ is the geodesic length function of a hyperbolic metric with geodesic boundary on $\Sigma_{g, r}$ if and only if

(a) for any two simple closed curves $a_{1}, a_{2}$ with $a_{1} \perp a_{2}$, let $a_{3}=a_{1} a_{2}$ and $b$ be the boundary of a regular neighborhood of $a_{1} \cup a_{2}$, then

$$
\begin{gathered}
t_{1} t_{2} t_{3}+2=t_{1}^{2}+t_{2}^{2}+t_{3}^{2}+t([b]), \\
t_{3}+t_{3}^{\prime}=t_{1} t_{2},
\end{gathered}
$$

where $t_{i}=2 \cosh \left(l\left(\left[a_{i}\right]\right) / 2\right)(i=1,2,3), t_{3}^{\prime}=2 \cosh \left(l\left(\left[a_{2} a_{1}\right]\right) / 2\right)$, and $t([b])=$ $2 \cosh (l([b]) / 2)$, and 


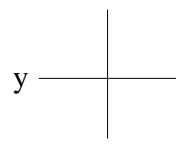

X

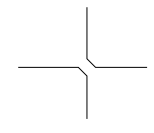

(a)

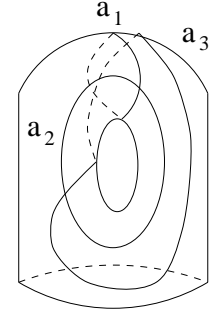

b

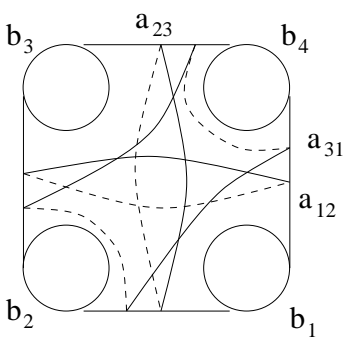

$\mathrm{a}_{31}=\mathrm{a}_{12} \mathrm{a}_{23}$ (b)

Figure 2

(b) for any two simple closed curves $a_{12}$ and $a_{23}$ with $a_{12} \perp_{0} a_{23}$, let $a_{31}=$ $a_{12} a_{23}$ and $b_{1}, b_{2}, b_{3}, b_{4}$ be the four boundary components of a regular neighborhood of $a_{12} \cup a_{23}$ so that $a_{i j}$, $b_{i}$ and $b_{j}$ bound a subsurface $\Sigma_{0,3}(i, j \leq 3)$, then

$$
\begin{gathered}
t_{12} t_{23} t_{31}+4=t_{12}^{2}+t_{23}^{2}+t_{31}^{2}+t_{12}\left(t_{1} t_{2}+t_{3} t_{4}\right)+t_{23}\left(t_{2} t_{3}+t_{1} t_{4}\right) \\
+t_{31}\left(t_{3} t_{1}+t_{2} t_{4}\right)+t_{1}^{2}+t_{2}^{2}+t_{3}^{2}+t_{4}^{2}+t_{1} t_{2} t_{3} t_{4}, \\
t_{31}+t_{31}^{\prime}=t_{12} t_{23}-t_{1} t_{3}-t_{2} t_{4},
\end{gathered}
$$

where $t_{i}=2 \cosh \left(l\left(\left[b_{i}\right]\right) / 2\right), t_{i j}=2 \cosh \left(l\left(\left[a_{i j}\right]\right) / 2\right)$, and $t_{31}^{\prime}=2 \cosh \left(l\left(\left[a_{23} a_{12}\right]\right) / 2\right)$.

Figure 2 (surfaces have the right-hand orientations in the front face) shows the location of these curves. Note that the curves $a_{i}$ and $a_{i j}$ are symmetric in the sense that $\left[a_{i}\right]\left[a_{j}\right]=\left[a_{k}\right]$ and $\left[a_{i j}\right]\left[a_{j k}\right]=\left[a_{k i}\right]$ for $k \neq i, j$ and $(i, j)=(1,2),(2,3),(3,1)$.

Relations (1), $\left(1^{\prime}\right),(2)$, and $\left(2^{\prime}\right)$ come from trace identities for $\operatorname{SL}(2, \mathbf{R})$ matrices.

Thurston's compactification of the Teichmüller space $T(\Sigma)$ (see [Bo], [FLP], [Th]) uses the embedding $\pi: T(\Sigma) \rightarrow \mathbf{R}^{\mathcal{S}(\Sigma)}$ sending $m$ to $l_{m}$. The theorem gives a complete description of the image of the embedding.

The proof of the theorem also shows the following result. Given a subset $F$ of $\mathcal{S}(\Sigma)$, let $\pi_{F}: T(\Sigma) \rightarrow \mathbf{R}^{F}$ be the map $\pi_{F}(m)=\left.l_{m}\right|_{F}$.

Corollary. (a) For a surface $\Sigma_{g, r}$ of negative Euler number and $r>0$, there exists a finite subset $F$ in $\mathcal{S}\left(\Sigma_{g, r}\right)$ consisting of $6 g+3 r-6$ elements so that the map $\pi_{F}: T\left(\Sigma_{g, r}\right) \rightarrow \mathbf{R}^{F}$ is a real analytic embedding onto an open subset which is defined by a finite set of (explicit) real analytic inequalities in the coordinates of $\pi_{F}$.

(b) For a surface $\Sigma_{g, 0}$ of negative Euler number, there exists a finite subset $F$ of $\mathcal{S}\left(\Sigma_{g, 0}\right)$ consisting of $6 g-5$ elements so that $\pi_{F}: T\left(\Sigma_{g, 0}\right) \rightarrow \mathbf{R}^{F}$ is an embedding whose image in $\mathbf{R}^{F}$ is defined by one real analytic equation and finitely many (explicit) real analytic inequalities in the coordinates of $\pi_{F}$.

It is shown by $\mathrm{S}$. Wolpert [Wo] that the number $6 g-5$ in part (b) of the corollary is minimal. The corollary without the statements about the image of the embedding was obtained previously by Okumura [Ok], Schmutz [Sc], and Sorvali [So]. Okumura [Ok1] has recently proven the corollary using a different method.

Some examples of the collection $F$ and the images of the Teichmüller spaces are as follows. For $\Sigma_{2,0}$, take $F=\left\{\left[a_{1}\right],\left[a_{2}\right],\left[a_{3}\right],\left[a_{4}\right],\left[a_{5}\right],\left[a_{6}\right],\left[a_{7}\right]\right\}$ as in Figure 3 . Then 

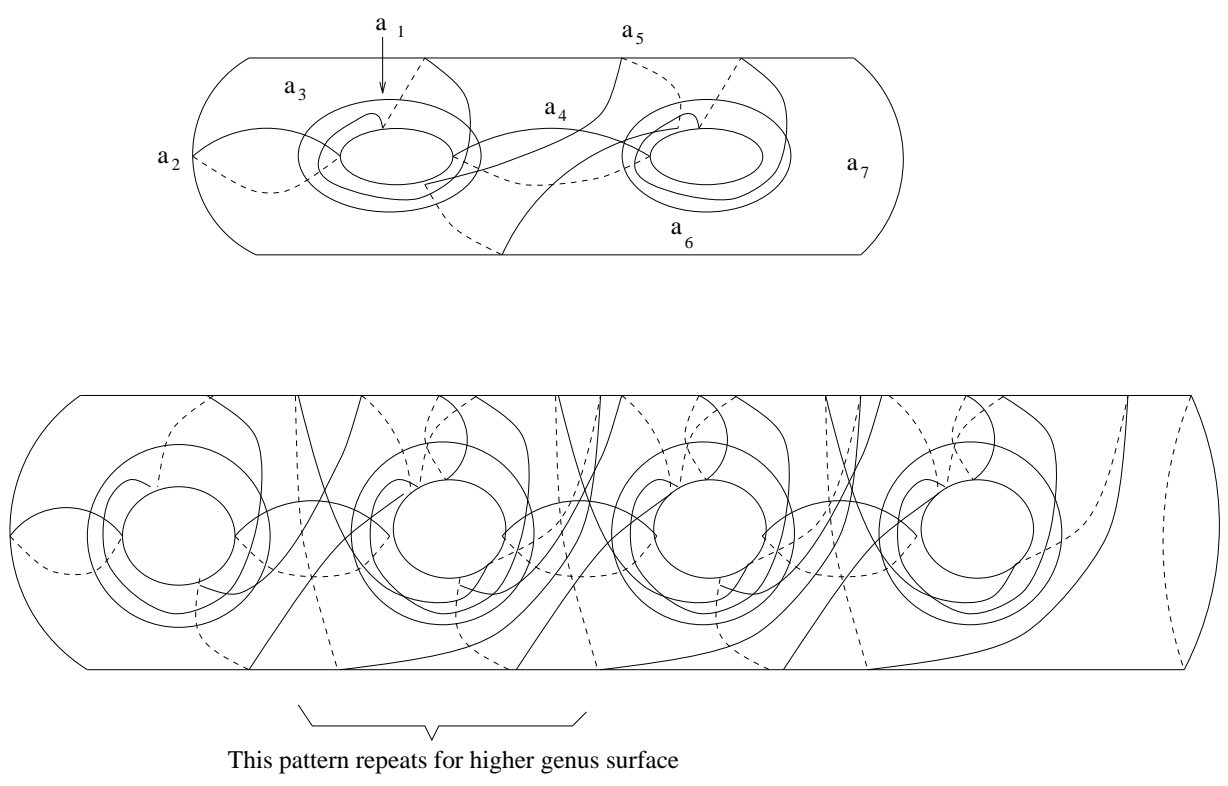

Figure 3

the map $\pi_{F}$ is an embedding with image $\pi_{F}\left(T\left(\Sigma_{2,0}^{0}\right)\right)=\left\{\left(t_{1}, t_{2}, t_{3}, t_{4}, t_{5}, t_{6}, t_{7}\right) \in\right.$ $\mathbf{R}_{>2}^{6} \mid t_{8}>2, t_{9}>2, t_{8}=t_{6} t_{7} t_{9}-t_{6}^{2}-t_{7}^{2}-t_{9}^{2}+2$, where $t_{8}=t_{1} t_{2} t_{3}-t_{1}^{2}-t_{2}^{2}-t_{3}^{2}+2$, and $\left.\left(2+t_{2}^{2}+t_{8}\right) t_{9}^{2}+2 t_{2}\left(t_{4}+t_{5}\right) t_{9}+2 t_{2}^{2}+t_{4}^{2}+t_{5}^{2}+t_{8}^{2}+t_{2}^{2} t_{8}-t_{4} t_{5} t_{8}-4=0\right\}$.

Remark. The theorem and the corollary hold for hyperbolic metrics with cusp ends (see [Lu1]).

\section{ACKNOWLEDGMENT}

I would like to thank F. Bonahon for calling my attention to several references. This work is supported in part by the NSF.

\section{§2. Sketch of the proof of the theorem}

There are several basic steps involved in the proof of the theorem. The proof is currently quite long. Below, we shall describe briefly the idea of the proof for a compact surface with non-empty boundary.

Step 1. We prove the result stated in Example 2 for $\Sigma_{0,4}$. This is essentially a careful application of the Maskit combination theorem [Mas] together with the trace relations for $\mathrm{SL}(2, \mathbf{R})$ matrices.

Step 2. We prove a gluing lemma. The classical gluing lemma for surfaces is as follows. Take two hyperbolic surfaces $X$ and $Y$ with geodesic boundary so that they have the same lengths at two boundary components $b_{X}$ and $b_{Y}$. Then one glues $X$ to $Y$ along $b_{X}$ and $b_{Y}$ by an isometry. However, the isometry is not unique due to the rotational symmetry of $S^{1}$. One obtains the so-called Fenchel-Nielsen twisting parameter for the gluing. We propose another procedure of gluing which will eliminate the parameter. Thus the result of the gluing produces a unique hyperbolic metric up to isotopy. The basic idea is as follows. Given a compact surface $\Sigma$, we decompose it as a union of two compact connected incompressible 


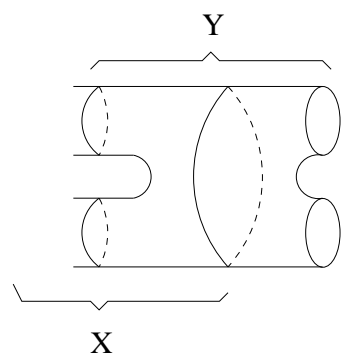

(a)

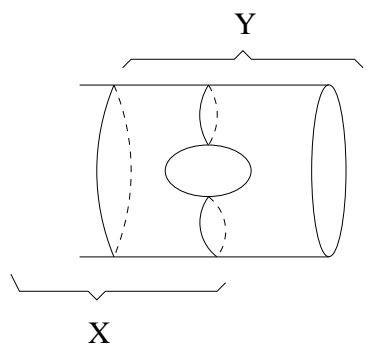

(b)

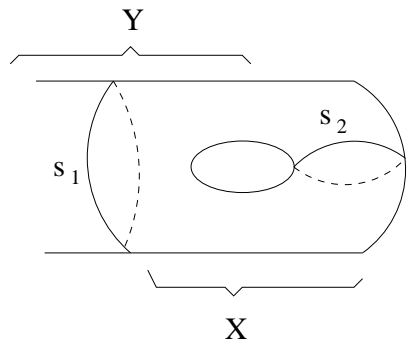

(c)

Figure 4

(meaning $\pi_{1}$-injective) subsurfaces $X$ and $Y$ so that $X \cap Y$ is homeomorphic to $\Sigma_{0,3}$ (see Figure 4). Let the three boundary components of $X \cap Y$ be $a_{1}, a_{2}$ and $a_{3}$. Then the gluing lemma states that for each hyperbolic metric $m_{X}$ and $m_{Y}$ on $X$ and $Y$ respectively so that $a_{i}$ are geodesics in both metrics with $l_{m_{X}}\left(a_{i}\right)=$ $l_{m_{Y}}\left(a_{i}\right), \quad(i=1,2,3)$ there is a hyperbolic metric $m$ in $\Sigma$ unique up to isotopy so that the restriction of $m$ to $X$ is isotopic to $m_{X}$ and the restriction of $m$ to $Y$ is isotopic to $m_{Y}$. The proof of the lemma is evident from the definition. For simplicity, we shall call this the gluing along 3-holed sphere lemma.

Step 3. We use the gluing along 3-holed sphere lemma to understand the hyperbolic metrics on $\Sigma_{1,2}$ by decomposing $\Sigma_{1,2}$ as a union $X \cup Y$, where $X \cong \Sigma_{1,1}$ and $Y \cong \Sigma_{0,4}-\left(b_{1} \cup b_{2}\right)$, where $b_{1}$ and $b_{2}$ are the two boundary components. See Figure 4(c). A slight generalization of the version of the gluing lemma stated above is needed to take care of the non-compact $Y$.

Step 4. We set up the induction procedure as follows. Define the norm of a surface $\Sigma_{g, r}$ to be $3 g+r$. Given a surface $\Sigma_{g, r}$, if $r \geq 2$, then $\Sigma_{g, r}=X \cup Y$, where $X=\Sigma_{g, r-1}$ and $Y=\Sigma_{0,4}$, and if $r=1, \Sigma_{g, 1}=X \cup Y$, where $X=\Sigma_{g-1,2}$ and $Y=\Sigma_{1,2}$ with $X \cap Y \cong \Sigma_{0,3}$ (see Figure 4). Thus given a function $f$ from $\mathcal{S}\left(\Sigma_{g, r}\right)$ to $\mathbf{R}_{>2}$ satisfying conditions (1), ( $\left.1^{\prime}\right),(2)$, and $\left(2^{\prime}\right)$, we consider the restrictions of $f$ to $\mathcal{S}(X)$ and $\mathcal{S}(Y)$ (both are viewed as subsets of $\mathcal{S}(\Sigma)$ under the inclusion maps). By the induction hypothesis, there exist hyperbolic metrics $m_{X}$ and $m_{Y}$ on $X$ and $Y$, respectively, so that $\left.f\right|_{\mathcal{S}(X)}=t_{m_{X}}$ and $\left.f\right|_{\mathcal{S}(Y)}=t_{m_{Y}}$. Now by the gluing lemma, there exits a metric $m$ on $\Sigma$ whose restriction to the two subsurfaces $X$ and $Y$ are isotopic to $m_{X}$ and $m_{Y}$. Thus, we have constructed a hyperbolic metric $m$ on $\Sigma$ so that $f$ is equal to $t_{m}$ on the subset $\mathcal{S}(X) \cup \mathcal{S}(Y)$ of $\mathcal{S}(\Sigma)$.

Step 5. This is the key step in the proof. The goal is to show that the above condition $\left.f\right|_{\mathcal{S}(X) \cup \mathcal{S}(Y)}=\left.t_{m}\right|_{\mathcal{S}(X) \cup \mathcal{S}(Y)}$ implies $f=t_{m}$.

Our observation is that the equations $\left(1^{\prime}\right)$ and $\left(2^{\prime}\right)$ give rise to an iteration process.

This shows that the value of $f$ at $\beta \alpha$ is determined by the values of $f$ at $\alpha, \beta$, $\alpha \beta$ in case $\alpha \perp \beta$, and the values of $f$ at $\alpha, \beta, \alpha \beta$ and $b_{s}$ 's in case $\alpha \perp_{0} \beta$. For instance, to determine $f$ on $\mathcal{S}^{\prime}\left(\Sigma_{1,1}\right)$, it suffices to know the value of $f$ at three vertices of an ideal triangulation in the modular configuration since the iteration equation $\left(1^{\prime}\right)$ will take care of the values of $f$ on $\mathcal{S}^{\prime}$.

We shall illustrate the proof of this major step by considering the special case of $\Sigma=\Sigma_{0,5}$. Take two disjoint, essential, non-boundary parallel, non-homotopic 


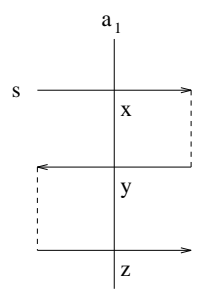

(a)

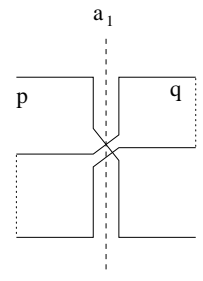

(b)

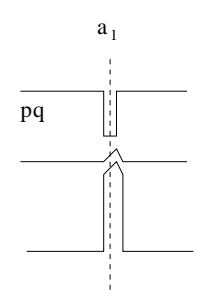

(c)

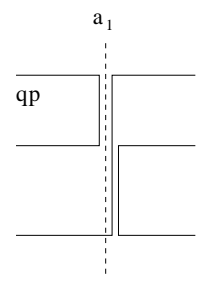

(d)

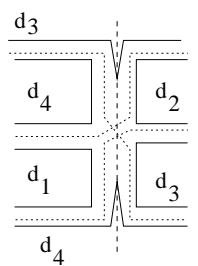

(e)

Figure 5

simple closed curves $a_{1}$ and $a_{2}$ in $\Sigma$. They give rise to a gluing along a 3-holed sphere decomposition of $\Sigma$, where we take $X_{i}$ to be the 4-holed sphere bounded by $a_{i}$ in $\Sigma$.

Now the given condition on $f$ and $t_{m}$ states that $f([a])=t_{m}([a])$ for all simple closed curves $a$ which are disjoint from either $a_{1}$ or $a_{2}$. A lemma of Lickorish below shows that if $f([a])=t_{m}([a])$ holds for all simple closed curves $a$ which intersect each $a_{i}$ in at most two points, then $f=t_{m}$.

Lemma (Lickorish). Suppose $s$ is an essential simple closed curve which intersects one of the two curves $a_{i}$ in at least three points. Let the norm of a curve $c$ be $\left|c \cap a_{1}\right|+\left|c \cap a_{2}\right|$. Then there exist two simple closed curves $p$ and $q$ with $p \perp_{0} q$ so that $s=p q$ and the norms of the curves $p, q$, qp, and each of the four boundary components of a regular neighborhood $N(p \cup q)$ of $p \cup q$ are less than the norm of $s$.

We sketch the proof of this lemma as follows. Suppose for definiteness that $\left|s \cap a_{1}\right|>2$. Then since $s$ is a separating simple closed curve, the intersection points of $s \cap a_{1}$ have alternating intersection signs in $a_{1}$. Pick up three adjacent intersection points $x, y$ and $z$ in $a_{1}$ and fix an orientation on $s$. Without loss of generality, we may assume that the arc from $x$ to $y$ (in curve $s$ ) in the orientation does not contain the point $z$. Then choose simple closed curves $p$ and $q$ as indicated in Figure 5. One verifies that the $p, q$ and $\partial N(p \cup q)$ satisfy the condition in the lemma (see [Lu2] for more details on curves in surfaces).

Thus, to finish the proof of the theorem, by the lemma above and the iterate equation $\left(2^{\prime}\right)$, it suffices to show that $f([a])=t_{m}([a])$ where $a$ intersects each $a_{i}$ at two points. There are only finitely many such $a$ up to homeomorphisms of the surface leaving each $a_{i}$ invariant. We verify the last condition $f([a])=t_{m}([a])$ for $a \perp_{0} a_{i}(i=1,2)$ through iterated uses of the relations $(2)$ and $\left(2^{\prime}\right)$.

This finishes the sketch of the proof.

\section{REFERENCES}

[Bo] F. Bonahon, The geometry of Teichmüller space via geodesic currents, Invent. Math. 92 (1988), 139-162. MR 90a:32025

[De] M. Dehn, Papers on group theory and topology, J. Stillwell (ed.), Springer-Verlag, Berlin and New York, 1987. MR 88d:01041

[FK] R. Fricke and F. Klein, Vorlesungen über die Theorie der Automorphen Funktionen, Teubner, Leipizig, 1897-1912.

[FLP] A. Fathi, F. Laudenbach, and V. Poenaru, Travaux de Thurston sur les surfaces, vol. 6667, Astérisque, Société Mathématique de France, 1979. MR 82m:57003

[Ke] L. Keen, Intrinsic moduli on Riemann surfaces, Ann. Math. 84 (1966), 405-420. MR 34:2859 
[Li] R. Lickorish, A representation of orientable combinatorial 3-manifolds, Ann. Math. 72 (1962), 531-540. MR 27:1929

[Lu1] F. Luo, Geodesic length functions and Teichmüller spaces, preprint.

[Lu2] Non-separating simple closed curves in a compact surface, Topology, in press.

[Mas] B. Maskit, On Klein's combination theorem, Trans. Amer. Math. Soc. 131 (1968), 32-39. MR 36:6618

[Ok] Y. Okumura, On the global real analytic coordinates for Teichmüller spaces, J. Math. Soc. Japan 42 (1990), 91-101. MR 90k:32071

[Ok1] _ Global real analytic length parameters for Teichmüller spaces, Hiroshima Math. J. 26 (1) (1996), 165-179. CMP 96:10

[Sc] P. Schmutz, Die Parametrisierung des Teichmüllerraumes durch geodätishe Längenfunktionen, Comment. Math. Helv. 68 (1993), 278-288. MR 94g:32028

[So] T. Sorvali, Parametrization for free Möbius groups, Ann. Acad. Sci. Fenn. 579 (1974), $1-12$.

[Th] W. Thurston, On the geometry and dynamics of diffeomorphisms of surfaces, Bul. Amer. Math. Soc. 19 (1988), 417-438. MR 89k:57023

[Wo] S. Wolpert, Geodesic length functions and the Nielsen problem, J. Diff. Geom. 25 (1987), 275-296. MR 88e:32032

Department of Mathematics, Rutgers University, New Brunswick, NJ 08903

E-mail address: fluo@math.rutgers.edu 